\title{
InfRaClavicular Brachial Plexus Block and Infusion for Treatment Of Long-Standing Complex Regional SyndRome Type 1: A CASE REPORT
}

\author{
Miles Day, MD, Ram Pasupuleti, MD, and Scott Jacobs, M.Ed, LPC
}

\begin{abstract}
Complex regional pain syndrome type 1 (CRPS 1 ) is a perplexing chronic pain condition that frustrates physicians and patients alike. The etiology of the condition resides in multiple theories and diagnosis can be difficult. Therapy focuses on pain management and restoration of physical function. Conservative treatment includes both non-pharmacological and pharmacological methods. Invasive therapy centers on sympathetic and somatic blocks and may evolve to neuromodulatory or neuraxial techniques.
\end{abstract}

In patients with severe pain and extrem- ity contractures with CRPS, often times the invasive blocks help in reduction of resting pain, but are ineffective in alleviating pain related to aggressive physical therapy. Continuous regional blockade with a percutaneous catheter is an effective, but seldom reported technique that can be used to treat both resting and movement related pain.

This presentation reviews the results of an infraclavicular brachial plexus infusion used to treat CRPS 1 that developed in a 49 year-old woman following a work-related upper extremity injury. The patient made a dramatic recovery with the infusion, which was maintained for two weeks. A T2-T3 sympathetic radiofrequency thermocoagulation was then performed to maintain pain relief and the infusion was stopped. The patient returned to work and has done well over the ensuing 19 months.

Keywords: Complex regional pain syndrome type 1, CRPS Type 1, Sympatheticallymaintained pain, neuromodulation, continuous regional blockade
Complex regional pain syndrome type 1 (CRPS 1; reflex sympathetic dystrophy) is a regional pain syndrome typically resulting from an extremity injury. Its hallmark is pain out of proportion to the initial injury. Associated findings include allodynia, vaso- and/or sudomotor changes, edema, and loss of extremity function (1). Treatment modalities are diverse and have varying rates of success. Though pain relief is a major goal in the treatment of CRPS, restoration of function and quality of life is equally, if not more important. We illustrate this in our presentation of a patient with an advanced case of CRPS-I of the right upper extremity with intolerable pain and almost total loss of function in the involved limb. Complete resolution of pain with full functional restoration was achieved following the placement of an infraclavicular brachial plexus catheter and infusion of local anesthetic and opi-

From International Pain Institute, Texas Tech University Health Sciences Center, Lubbock, Texas, and The Pain Institute, Louisville, Kentucky. Address Correspondence: Miles Day, MD, DABPM, FIPP, International Pain Institute, Texas Tech University Health Sciences Center, Lubbock, Texas 79413

E-mail: Miles.day@ttuhsc.edu

Support: Nothing of value received from a commercial entity related to this research

Conflict of Interest: None oid for a period of twelve days with concomitant aggressive in-patient, physical and occupational therapy.

\section{Case History}

A forty nine-year-old female presented to our clinic with a history of a work related injury when a heavy metal plate fell on her right forearm, leading almost immediately to pain and swelling in her forearm, wrist and hand. There was no evidence of bony injury on radiographic imaging, and when the swelling and pain did not diminish in two weeks, she was diagnosed with CRPS-I of the right upper extremity. The patient was seen by several pain physicians and treated with different modalities. Initial treatment consisted of opioids and neuropathic medications that provided no significant benefit. She then underwent multiple stellate ganglion blocks, T2-T3 sympathetic blocks with minimal and short-lived relief, an epidural catheter and finally implantation of a spinal cord stimulator (SCS). Paresthesias were felt in the area of the pain, but stimulation afforded no analgesia.

The patient presented to our clinic three years after the initial injury. She had swelling and pain in the right upper extremity from mid arm to the hand.
The pain was described as burning and sharp, with the intensity being a $10 / 10$ on the visual analog scale. Light touch, wind blowing across, and clothes touching the arm were all extremely painful. The patient was ambidextrous, with left being her dominant hand, but she used her right hand for writing and eating. After this injury the patient lost complete use of her right upper extremity and was placed on disability after being unable to work. She developed a claw-hand deformity and was at the point of wanting to have the arm amputated. The patient's medications included OxyContin ${ }^{\circledR} 20$ mg TID, hydrocodone/acetaminophen 10/325 TID, valdecoxib $20 \mathrm{mg}$ QD and alprazolam $0.5 \mathrm{mg}$ BID. A prior MRI of the right arm was negative for bone injury and an EMG of the right upper extremity was positive for carpal tunnel syndrome.

Examination revealed distinct circumferential edema in the right upper extremity extending form the midforearm to the hand (Figure 1). The skin appeared shiny and the nails were brittle with the nail beds appearing blanched. Significant allodynia to light touch and cold was noted. There was no evidence of hyperhidrosis or increased hair growth. The right hand had a claw 


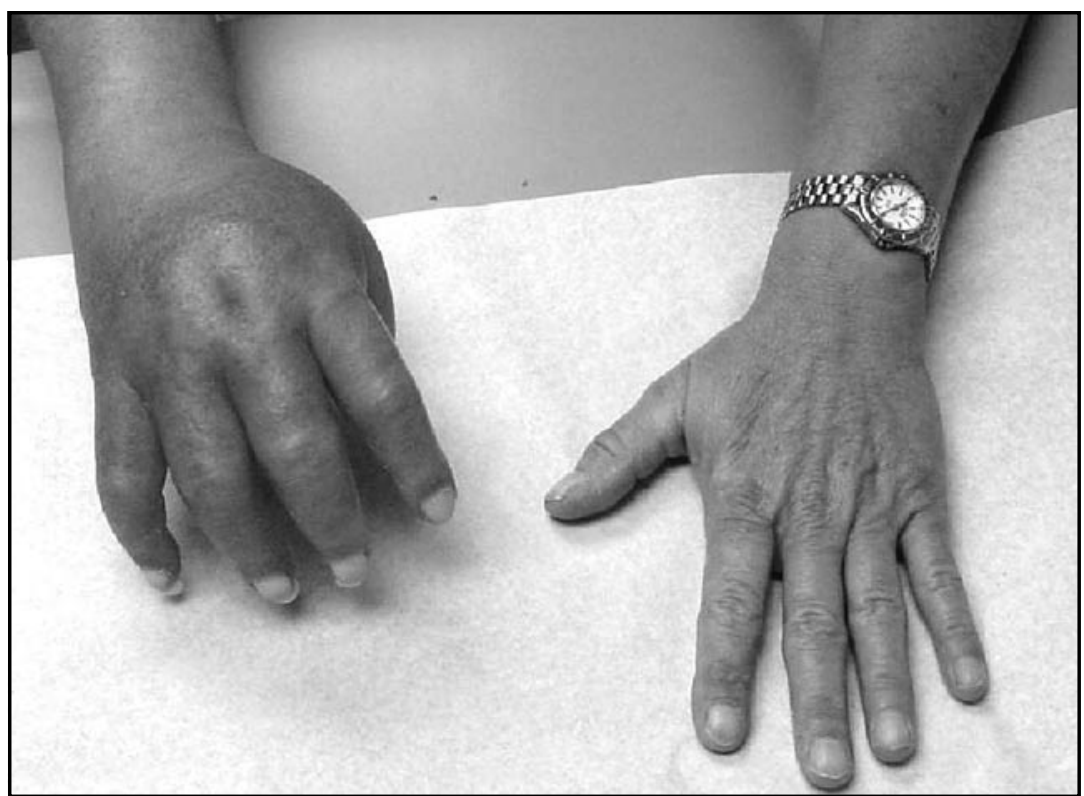

Fig 1. Pre-catheter photograph, illustrating the severe edema and blanching of the nail beds

Table 1. Raj technique

1. Patient supine with head turned away from the arm to be blocked

2. Abduct arm to 90 degrees

3. Identify and mark the entire length of the clavicle

4. Identify the midpoint of the clavicle and mark

5. Palpate the brachial artery in the arm and mark

6. Palpate and mark the ipsilateral C6 tubercle

7. Draw a line from the $\mathrm{C} 6$ tubercle to the brachial artery in the arm (it should go through the midpoint of the clavicle)

8. Physician should stand on the side opposite of the arm to be blocked

9. Raise a skin wheal 1 inch below the inferior border of the clavicle at its midpoint

10. Insert the block needle and catheter through the skin and aim towards the brachial artery using the line drawn in step 7 as a guide.

11. Stimulate and advance the block needle/catheter until motor stimulation is noted in the hand. Reduce the stimulation to a target of $0.5 \mathrm{~mA}$.

12. While maintaining motor stimulation, inject $1 \mathrm{ml}$ of local anesthetic. This should abolish the motor stimulation if the needle/catheter is appropriately placed. Contrast can also be injected under fluoroscopy to confirm proper placement.

13. Secure the catheter to the skin with nylon suture and steri-strips and cover with a sterile bandage.

14. The catheter can be bolused with local anesthetic on a daily basis or a continuous infusion of local anesthetic/opioid can be initiated. deformity with very minimal movement at the wrist, metacarpo-phalangeal and inter-phalangeal joints. Movement was also limited at the elbow and shoulder, with both joints being held in a flexed position. Right upper extremity strength was $2 / 5$ for forearm flexion and $1 / 5$ for forearm extension, wrist flexion/ extension, and finger flexion/extension. Trophic changes were evident in the upper forearm.

Due to the severity and ongoing nature of the patient's CRPS I, she was admitted to the hospital and a brachial plexus catheter was placed in the right upper extremity using an infraclavicular approach described by Raj et al (Table 1) (2). An infusion consisting of $0.1 \%$ ropivacaine and $5 \mathrm{mcg} / \mathrm{cc}$ of fentanyl was initiated through the catheter at a rate of $12 \mathrm{cc}$ per hour. The patient reported a small amount of pain relief and was able to initiate minimal movements in her right hand. On post-operative day 1 , the concentration of the ropivacaine was increased to $0.15 \%$, which improved the analgesia. Late that day the catheter was accidentally dislodged and subsequently replaced using the same technique. The patient noted improved analgesia, and improvement in the edema and movement of the right hand and wrist with the second catheter. During the ensuing two-week hospital admission, the infusion was continued with institution of aggressive physical and occupational therapy. The function in the right hand improved gradually with dramatic pain relief and decrease in the swelling (figure 2 and 3 ). By the end of ten days, the patient was able to pick up a pen and write, and feed herself with her right hand. The patients' oral medications were gradually weaned as her analgesia and functional status improved.

A right T2-T3 sympathetic radiofrequency thermocoagulation was performed on the thirteenth day of admission and the catheter was removed late the same day. There was no evidence of allodynia, pain or swelling at the time of her discharge on the fourteenth day. The patient returned to work immediately after discharge and weekly telephone followups for six weeks showed no evidence of swelling or pain. In a telephone interview 19 months later, the patient continues to work and stated that she is $75 \%$ better. Her pain level is a $4 / 10$ on the VAS and she 


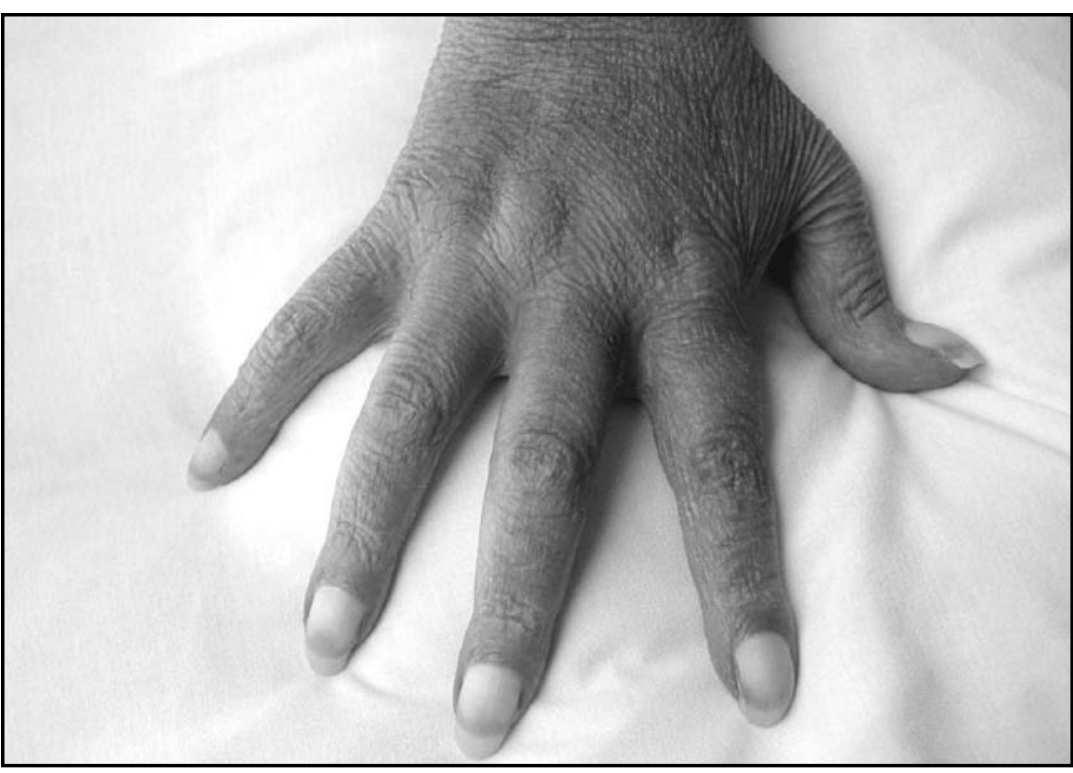

Fig 2. Outstretched hand at time of discharge with resolution of the edema, with uniformly blanched nail beds

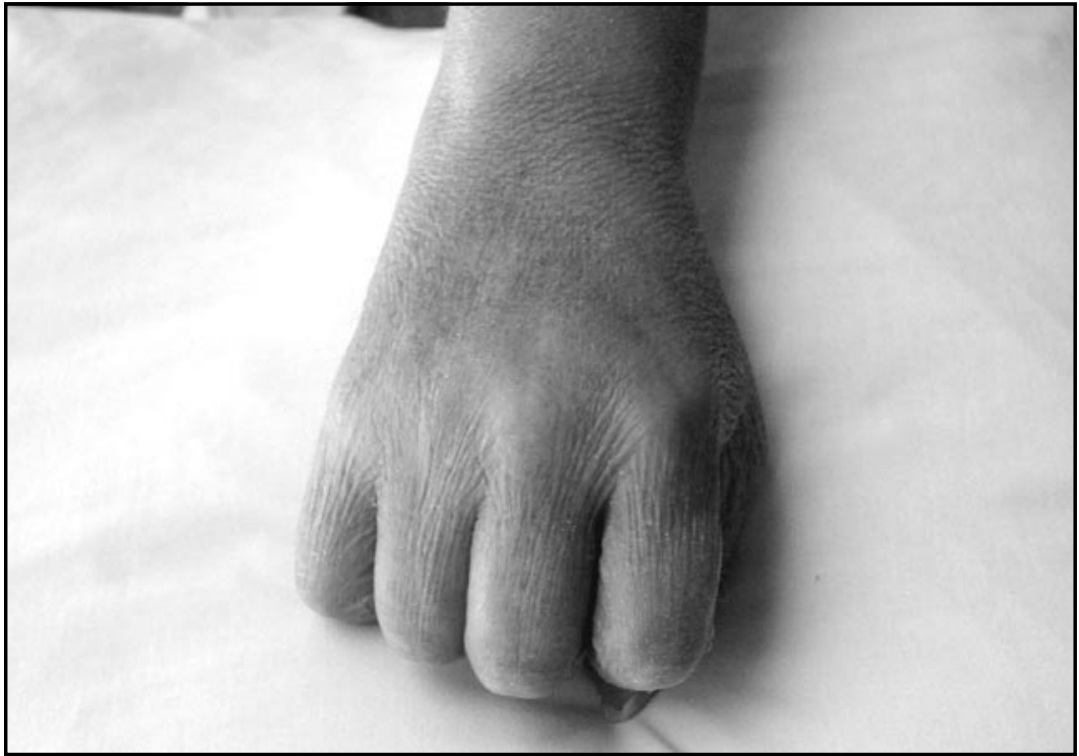

Fig. 3. Closed fist at time of discharge. The patient could not perform this maneuver at the time of admission

takes tizanidine $4 \mathrm{mg}$ TID and valdecoxib $10 \mathrm{mg}$ BID for pain. She no longer uses her cervical SCS.

\section{DISCUSSION}

In 1994, the International Association for the Study of Pain met to discuss the taxonomy for reflex sympathetic dystrophy (RSD) and causalgia. As a result of the meeting, the term complex regional pain syndromes (CRPS) was devised with RSD and causalgia becoming CRPS- ing in neuroma formation with spontaneous discharge, ephaptic transmission between an injured, afferent nociceptor and an adjacent efferent sympathetic nerve, and an alteration in the gate theory of pain. Centrally, the peripheral injury can result in a perpetual state of hyperexcitability in spinal cord neurons. Regardless of the mechanism, the end result is a painful syndrome.

Pain out of proportion to the original injury is the most common complaint and this can be associated with decreased use of the affected limb. Physical findings include autonomic dysregulation (edema, hyperhidrosis, alterations in blood flow), sensory abnormalities (hyper-/hypoesthesia, allodynia to cold and mechanical stimuli), motor dysfunction (weakness, tremor, joint stiffness), and trophic changes (brittle nails, altered hair growth, glossy skin, muscle atrophy) (6). Psychological disturbances such as anxiety, hopelessness and depression may also be present.

Diagnostic studies are helpful in confirming the diagnosis of CRPS. These include plain $\mathrm{x}$-rays, triple-phase scintigraphy, thermography, nerve conduction tests, electromyograms, and tests of sudomotor function. It is important to remember that a single positive or negative test does not rule in nor rule out CRPS. Instead they should be interpreted along with other tests and the clinical evaluation to make the diagnosis.

The treatment for CRPS-I and II is similar. The interdisciplinary approach combining pain management, psychological treatment, and rehabilitation is favored over individual approaches (1). A variety of medications are used to alleviate pain and related maladies. These include nonsteroidal anti-inflammatory drugs, opioids, anti-epileptic drugs, skeletal muscle relaxants, antidepressants, NMDA-receptor antagonists, alpha-1 antagonists, alpha-2 agonists, and sleep enhancers. Interventional therapy ranges from minimally invasive to highly invasive. Minimally invasive blocks target sympathetic and somatic nerves and can be done single-shot or via a percutaneous catheter when prolonged analgesia is needed $(1,4,6,7)$. Highly invasive therapy includes placement of tunneled neuraxial catheters, peripheral nerve / spinal cord stimulation and intrathecal drug delivery $(1,4,8)$. These therapies combined with rehabilitation and psycholog- 
ical counseling seek to provide analgesia and restoration of function.

An infraclavicular brachial plexus catheter with a continuous infusion of local anesthetic and opioid was chosen for this patient secondary to the ongoing, debilitating nature of her CRPS-I in the right arm. She required an intervention that could provide analgesia for both the sympathetic and somatic components of her pain.

Labat first described the infraclavicular approach to the brachial plexus in 1922 (9). Raj et al (2) published a modification of the Labat technique in 1973, and several authors (9-11) have published modifications of Raj et al's technique since. Advantages of the infraclavicular approach for catheter placement in the brachial plexus over other techniques include ease of threading of the catheter, maintenance of a sterile environment around the catheter insertion site, lower incidence of catheter dislodgement with passive or active upper extremity movement, and avoidance of most of the theoretical complications of supraclavicular techniques (pneumothorax, phrenic nerve block, intravertebral artery injection, and extraand intradural injection) (12).

Despite a lengthy literature search, only a few papers were identified describing this technique to treat CRPS. Murray and Atkinson (13) reported on the placement of an axillary brachial plexus catheter with local anesthetic infusion in a patient with CRPS-I of the left upper extremity. They placed a series of 3 catheters for durations of 24-48 hours at approximately 2 -week intervals. She was able to achieve near complete analgesia and full use of her left arm. The benefit was sustained at a 2-month follow-up.

Ribbers et al (14) performed a prospective, non-placebo controlled study involving the placement of axillary plexus blockade catheters for 6 patients with CRPS-I of an upper extremity. Each of the patients received varying numbers of "sessions" of brachial plexus blockade with either continuous infusion or intermittent boluses, and physical therapy. A "session" consisted of a 2-week treatment with a brachial plexus catheter and an ensuing 1-week rest period. Three of the patients were poor responders. Each of these patients had stage 3 (atrophic phase) CRPSI. The remaining 3 patients (one with stage 1 and two with stage 2 CRPS-I) responded well with lasting improvement in pain as well as functional status in the affected extremity. They concluded that this treatment modality should be furthered evaluated for early stage CRPS-I.

Wang et al (15) published a case report on the use of an axillary brachial plexus block with patient-controlled analgesia for stage 1 CRPS-I of a right upper extremity. Their patient responded favorably after a 7-day infusion with concomitant physical therapy.

While a brachial plexus catheter is not a new treatment modality, it does appear its use to treat refractory CRPS pain and debility is either underutilized or just infrequently reported based on the sparsity of literature regarding its use. Our institution has used continuous peripheral nerve blockade on many occasions when treating severe cases of CRPS with varying degrees of success in providing pain relief and restoring functional status of the affected extremity.

Author Affiliation:
Miles Day, MD
Assistant Professor
Pain Management/Anesthesiology
Co-Director Pain Services
International Pain Institute
Texas Tech University Health Sciences
Center
Lubbock, Texas 79413
E-mail: Miles.day@ttuhsc.edu
Ram Pasupuleti, MD
The Pain Institute
252 Whittington Parkway
Louisville, Kentucky
E-mail: ram@thepianinstitute.com
Scott Jacobs, M.Ed, LPC
International Pain Institute
Texas Tech University Health Sciences
Center
Lubbock, Texas 79413

\section{Conclusion}

This case report illustrates the success of infraclavicular brachial plexus infusion in returning a patient to the working society who at one point considered amputation as a means of getting relief.

\section{RefERENCES}

1. Stanton-Hicks M, Burton A, Carr D, et al. An updated interdisciplinary clinical pathway for CRPS: report of an expert panel. Pain Practice 2002; 2:1-16.

2. Raj P, Montgomery S, Nettles D, et al. Infraclavicular brachial plexus block-a new approach. Anesth Analg 1973; 52:897-903.

3. Merskey H, Bogduk N. Classification of Chronic Pain: Descriptions of Chronic Pain Syndromes and Definitions of Pain Terms, 2nd ed. Seattle, IASP Press, 1994.

4. Raj P. Complex regional pain syndromes reflex sympathetic dystrophy and causalgia. Curr Rev Pain 1998; 2:242-253.

5. Stanton-Hicks M. Reflex sympathetic dystrophy: A sympathetically mediated pain syndrome or not. Curr Rev Pain 2000; 4: 268-275.

6. Manning D, Loar C, Raj P et al. Neuropathic Pain. In Raj P (ed). Pain Medicine: A Comprehensive Review 2nd ed. St. Louis, Mosby, 2003, pp 77-93.

7. Ramamurthy S, Hoffman J. Intravenous regional guanethidine in the treatment of reflex sympathetic dystrophy/causalgia: a randomized, double-blind study. Anesth Analg 1995; 81:718-723.

8. Kumar K, Nath R, Toth C. Spinal cord stimulation is effective in the management of reflex sympathetic dystrophy. Neurosurgery 1997; 40:503-509.

9. Borgeat A, Ekatodramis G, Dumont C. An evaluation of the infraclavicular block via a modified approach of the Raj technique. Anesth Analg 2001; 93:436-441.

10. Sims J. A modification of landmarks for infraclavicular approach to brachial plexus block. Anesth Analg 1977; 56:554-555.

11. Whiffler K. Coracoid block-a safe and easy technique. Br J Anaesth 1981; 53:845-848.

12. Raj P. Infraclavicular approaches to brachial plexus anesthesia. Tech Reg Anesth Pain Manage 1997; 1:169-177.

13. Murray P, Atkinson R. Continuous axillary brachial plexus blockade for reflex sympathetic dystrophy. Anaesthesia 1995; 50: 633-635.

14. Ribbers G, Geurts A, Rijken R et al. Axillary brachial plexus blockade for the reflex sympathetic dystrophy syndrome. Int / Rehabil Res 1997; 20:371-380.

15. Wang L, Chen H, Chang P et al. Axillary brachial plexus block with patient controlled analgesia for complex regional pain syndrome type I: A case report. Reg Anesth Pain Med 2001; 26:68-71. 